\title{
Microscopy Characterization of Aqueous PVAC Glue Penetration in Double Coated Paperboard Systems
}

\author{
D. M. Williams, ${ }^{*}$ B. Ninness, ${ }^{* *}$ D. Ventresca, ${ }^{* *}$ G. W. Welsch ${ }^{* *}$ \\ *Analytical Sciences, 1897 Bldg. The Dow Chemical Company, Midland, MI 48667 \\ ** Styron LLC, 1604Bldg. Midland, MI 48667
}

Gluing together coated beverage packaging and folding cartons necessitates that the liquid-phase portion of the glue be able to penetrate the coating layer, and also the backside of the underlying fiber substrate, in order to dewater the glue, set it and form the final bond. An attempt was made to quantify the extent of aqueous glue penetration into the coating layers using both optical and electron microscopy techniques utilizing tracers in the glue.

Small $(<25 \mathrm{~nm})$, monodispersed metallic oxide particles were investigated as tracer materials for the aqueous glue. Backscatter electron SEM images of the cross sections revealed no migration of the nanoparticles (Figure 1). It is believed that upon drying the $25 \mathrm{~nm} \mathrm{CeO}_{2}$ particles aggregate into clusters that bridged the $100-1000 \mathrm{~nm}$ surface pores (measured by $\mathrm{Hg}$ porisimetry), resulting in the filtercake observed. Although the metal oxide tracer SEM-EDS technique did not allow us to monitor aqueous-glue penetration, the refinement of this method may prove valuable to other research work in the coating area. The small nanoparticles of metallic oxides demonstrate strong and unique X-ray emission lines even when present at very low concentrations.

The aqueous glue was stained with Rhodamine-B dye, building on techniques described by Ozaki et al [1]. Images were obtained on polished cross-sections using a Confocal Laser Scanning Microscope. Sample preparation proved to be quite critical in obtaining artifact-free images and in maintaining the integrity of the glued samples. Polishing by microtome without embedding the sample in epoxy gave the best results (Figure 2). Since the same amount of glue was applied, using the same glue application technique, the only difference in glue layer thickness visualized in these images should be due to the differing degrees of glue penetration. It is evident from the images and their respective measurements that the double-coated samples with an Air Knife applied topcoat exhibited much less penetration of glue into the coating. The typical thickness of the dried glue layer for the Blade/Blade samples was roughly $25-30 \mu \mathrm{m}$, with the composite coating/glue structure to be roughly $50-100 \mu \mathrm{m}$ thick. Measurements from the fluorescent imaging indicated that a portion of the aqueous glue penetrated completely through the coating and into the fiber substrate.

Dark field optical microscopy of an ion polished sample confirmed the Rhodamine-B dye effectively migrated from the applied glue layer, through the backside and topside coatings, and entered the fiber substrate (Figure 3). The research area encompassing glue setting and penetration is still evolving, however we see great utility in the continuing improvement of analytical microscopy techniques that allow the penetration of the glue to be monitored and quantified. 


\section{References}

[1] Ozaki, Y., et al., Journal of Pulp Paper Sci, (2005), pp. 48 - 52.
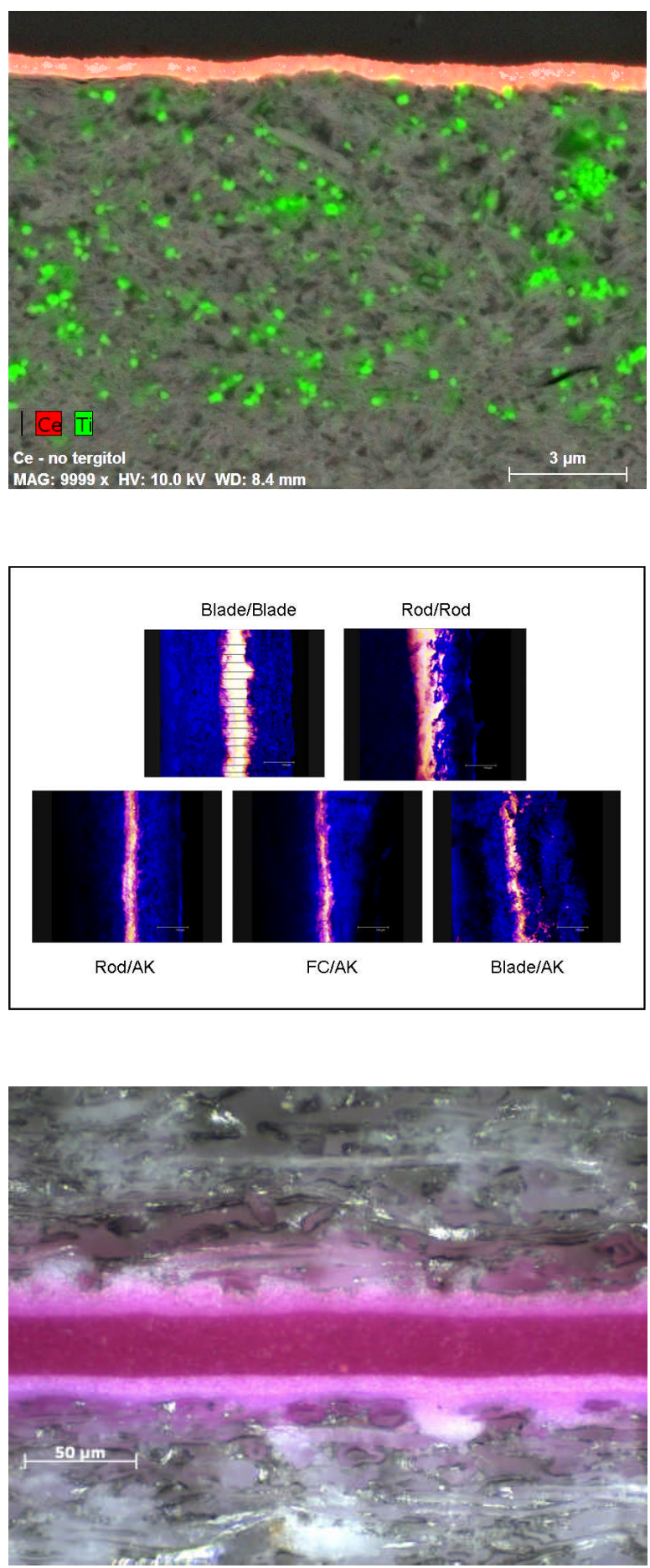

FIG. 1. SEM elemental map of the Blade/Blade coated sample with the $\mathrm{CeO}_{2}$ glue dispersion (red) applied to the surface. The $\mathrm{CeO}_{2}$ nano-particles produced a uniform and dense layer at the surface of the coating, with no indication that the small particles entered the coating. The delineation of the base and top coating layers is apparent by $\mathrm{TiO}_{2}$ (green) in the topcoat. The image horizontal field width $=18 \mathrm{um}$

FIG. 2. Confocal Laser Scanning Microscope images of microtome polished glued double coated application systems. The glue layer, and/or any portion of the glue vehicle which contains the Rhodamine dye are bright. In an attempt to quantify the degree of glue penetration measurements were taken on each image, as illustrated by the horizontal lines in the Blade/Blade image. Horizontal field width $=750 \mathrm{um}$.

FIG. 3. Optical microscopy image showing penetration of dye through coating layers into the base sheet fibers. This sample was prepared using ion beam polishing. Image horizontal field width $=300 \mathrm{um}$. 